\title{
Dose Optimization of the Administered Activity in Pediatric Bone Scintigraphy: Validation of the North American Consensus Guidelines
}

\author{
Karen L. Ayres, Stephanie E. Spottswood, Dominique Delbeke, Ronald Price, Pamela K. Hodges, Li Wang, \\ and William H. Martin \\ Department of Radiology and Radiologic Sciences, Vanderbilt Children's Hospital/Vanderbilt University Hospital, Nashville, \\ Tennessee
}

The 2010 North American Consensus Guidelines (NACG) for pediatric administered doses and the European Association of Nuclear Medicine (EANM) Dosage Card guidelines recommend lower activities than those administered at our institution. We compared the quality of the lower-activity images with the higher-activity images to determine whether the reduction in counts affects overall image quality. Methods: Twenty patients presenting to our pediatric radiology department for bone scintigraphy were evaluated. Their mean weight was $20 \mathrm{~kg}$. The patients were referred for oncologic $(n=10)$, infectious/inflammatory $(n=5)$, and pain $(n=5)$ evaluation. Dynamic anterior and posterior images were acquired for 5 min for each patient. Data were subsampled to represent different administered activities corresponding to the activities recommended by the NACG and the EANM Dosage Card. Images were evaluated twice, first for diagnostic quality and then for acceptability for daily clinical use. Results: There was no statistically significant difference in the diagnostic quality of the images from any of the 3 protocols. Pathologic uptake was correctly identified independent of the administered activity, although there was a single false-positive result for an EANM image. When images were subjectively evaluated as acceptable for daily clinical use, there was a slight preference for the higher-activity images over the NACG $(P=0.04)$. Conclusion: The recommended administered activities of the NACG produce images of diagnostic quality while reducing patient radiation exposure.

Key Words: North American Consensus Guidelines; dose reduction; pediatric bone scintigraphy

J Nucl Med 2015; 56:1391-1394

DOI: 10.2967/jnumed.115.156141

$\mathbf{R}$ adiation dose reduction is a major goal within the imaging community. The rate of use of ionizing radiation was quantified in a study analyzing the use of diagnostic ionizing radiation studies in over 350,000 children, selected on the basis of continuous enrollment in United Healthcare between 2005 and 2007 in 5 major markets including Dallas, Texas, and Orlando, Florida. Forty-three

Received Feb. 25, 2015; revision accepted Jul. 13, 2015.

For correspondence or reprints contact: Karen L. Ayres, Vanderbilt University Hospital, 1161 21st Ave. S., Medical Center North, Suite CCC1121, Nashville, TN 37232-2675.

E-mail: karen.ayres@vanderbilt.edu

Published online Jul. 23, 2015.

COPYRIGHT (C) 2015 by the Society of Nuclear Medicine and Molecular Imaging, Inc. percent of these children were exposed to radiation from at least one diagnostic imaging procedure performed over the 3-y observation period (1). Twenty-five percent of the children had at least 2 imaging procedures during that period, and $16 \%$ had at least 3 . Bone scintigraphy was the most commonly performed nuclear medicine procedure. These statistics are important to recognize because children are at greater risk of radiation side effects such as latent malignancy because of their rapid development and relatively long remaining life expectancy. On the basis of atomic bomb survivor data, the likelihood of solid tumor malignancy after exposure to ionizing radiation is $1.0-1.8$ times higher in a 10 -y-old child than in a young adult, and the risk of leukemia is approximately double (2). Because many children have multiple imaging procedures, cumulative exposure must also be considered.

In 2010, experts in nuclear medicine collaborated to produce the North American Consensus Guidelines (NACG) (3). The NACG were updated in 2014 with no change to the recommended bone scintigraphy administered activities (4). These guidelines provide recommendations for standardized administered activities for many common nuclear medicine studies performed on children, including bone scintigraphy, renography, and ${ }^{18} \mathrm{~F}-\mathrm{FDG}$ PET/CT. When compared with administered activities in many imaging institutions, including our own, the NACG-recommended activity for bone scintigraphy is much lower for all patient weights. The aim of the present study was to evaluate the quality of bone scintigraphy images using the administered activities recommended in the NACG, as compared with the body surface area-based activities administered at our institution. The NACG were also compared with the 2008 European Association of Nuclear Medicine (EANM) Dosage Card (equivalent to the since-released 2014 EANM Dosage Card).

Table 1 shows the administered activities and effective dose equivalents for children of different weights when the 3 different administered activity protocols are followed, highlighting the variability most notably in the smallest children. Data were derived from Publication 106 of the International Commission on Radiological Protection (5).

\section{MATERIALS AND METHODS}

Institutional Review Board approval for the project was granted as a quality assurance project, and the need for informed consent was waived. Twenty pediatric patients referred to our nuclear medicine department for bone scintigraphy were prospectively studied. The clinical indications included malignancy, infection/fever of unknown 
TABLE 1

Administered Activities and Effective Doses for 3 Dosing Schedules

\begin{tabular}{|c|c|c|c|}
\hline Weight (kg) & $\mathrm{MBq}$ & $\mathrm{mSv}$ & $\begin{array}{l}\text { Effective dose } \\
\text { reduction vs. } \\
\text { institutional }\end{array}$ \\
\hline \multicolumn{4}{|l|}{ Institutional } \\
\hline 5 & 148 & 7.8 & \\
\hline 10 & 207 & 5.6 & \\
\hline 20 & 370 & 5.1 & \\
\hline 30 & 444 & 5.0 & \\
\hline 40 & 570 & 5.5 & \\
\hline 50 & 666 & 5.4 & \\
\hline 60 & 696 & 4.7 & \\
\hline 70 & 740 & 4.2 & \\
\hline \multicolumn{4}{|l|}{ NACG } \\
\hline 5 & 46 & 2.5 & $-68 \%$ \\
\hline 10 & 93 & 2.5 & $-55 \%$ \\
\hline 20 & 185 & 2.6 & $-49 \%$ \\
\hline 30 & 278 & 3.1 & $-38 \%$ \\
\hline 40 & 270 & 3.6 & $-35 \%$ \\
\hline 50 & 463 & 3.8 & $-30 \%$ \\
\hline 60 & 555 & 3.7 & $-21 \%$ \\
\hline 70 & 648 & 3.7 & $-12 \%$ \\
\hline \multicolumn{4}{|c|}{ EANM Dosage Card } \\
\hline 6 & 60 & 3.2 & $-59 \%$ \\
\hline 10 & 95 & 2.6 & $-54 \%$ \\
\hline 20 & 170 & 2.4 & $-53 \%$ \\
\hline 30 & 240 & 2.7 & $-46 \%$ \\
\hline 40 & 310 & 3.0 & $-45 \%$ \\
\hline 50 & 375 & 3.0 & $-44 \%$ \\
\hline 60 & 445 & 3.0 & $-36 \%$ \\
\hline 68 & 490 & 2.8 & $-33 \%$ \\
\hline
\end{tabular}

EANM activities include patient weights of 6 and $68 \mathrm{~kg}$ rather than 5 and $70 \mathrm{~kg}$, based on design of EANM Dosage Card.

origin, and pain. The patients ranged from 2 mo to $16 \mathrm{y}$ old, with the exception of a patient who was 20 y old but within the pediatric weight for dosing $(50.5 \mathrm{~kg})$. Patient demographics are presented in Table 2.

The patients were administered our standard institutional activities of ${ }^{99 \mathrm{~m}} \mathrm{Tc}-m e t h y l e n e$ diphosphonate based on a body surface area nomogram (6). For all patient weights, our institutional activities were greater than the suggested activities based on the NACG and EANM Dosage Card. Dynamic anterior and posterior images of the chest were acquired for $5 \mathrm{~min}$ in 30-s frames using a low-energy, high-resolution, parallel-hole collimator with an energy window of $140 \mathrm{keV} \pm 10 \%$. The imaging time at our institution is $5 \mathrm{~min}$, as this provides between 500,000 and 1,000,000 counts, which falls within the recommendation of the Society of Nuclear Medicine and Molecular Imaging (7), and most patients can lie reasonably still for $5 \mathrm{~min}$. Images acquired over more than $5 \mathrm{~min}$ are subject to increased motion artifacts. The images were acquired approximately $2.5 \mathrm{~h}$ after administration of the radiopharmaceutical — an interval that is standard and also in line with the Society of Nuclear Medicine and Molecular Imaging guidelines.

The data were then subsampled to produce images simulating lower activities by summing several frames proportionate to the decreased activity. For example, when the NACG administered activity for a patient was $50 \%$ of the institutional administered activity, then $50 \%$ of the counts ( 5 of the 10 frames, representing 2.5 min of imaging time) were used to generate the NACG image. Percentages were rounded to the nearest $10 \%$. These reconfigured images were equivalent to images that would have been acquired over 5 min using the lower administered activities as per the different imaging protocols. Institutional, EANM, and NACG images were generated for each patient, as well as an arbitrary 1-min image, for a total of 80 images.

The images were evaluated independently by 2 experienced nuclear medicine physicians, including a pediatric nuclear medicine specialist, using the following subjective grading scale: 1, poor; 2, fair; 3, good; and 4, excellent. Images were presented one at a time, and the interpreters were masked to which imaging schedule was used to generate each image. The interpreters were asked to describe any pathologic uptake. Four of the 20 patients had pathologic findings in the chest, using contemporaneous diagnostic bone scintigraphy as the gold standard for the presence of visible findings.

At a second, separate, sitting, 2 experienced nuclear medicine physicians working in an unmasked fashion independently evaluated the images for acceptability for daily clinical practice. High- and lowdose images were compared side by side, with the interpreters aware of which images represented the NACG, EANM, institutional, and 1-min images. While evaluating all 4 images for a given patient, the interpreters were asked which of the images were subjectively acceptable for daily clinical use.

The statistical likelihood of producing an image of diagnostic quality was compared among the different administered activity strategies using the Pearson $\chi^{2}$ test. The Pearson test was also applied to the designation of acceptable or not for daily clinical use. There were not enough patients with pathologic findings within the included field of view to apply statistical analysis to this evaluation.

TABLE 2

Patient Demographics and Indications for Imaging

\begin{tabular}{lc}
\hline \multicolumn{1}{c}{ Demographic } & Data \\
\hline Sex $(n)$ & 10 \\
Female & 10 \\
Male & \\
Age & mo-16 ${ }^{\star}$ \\
Range & $7.1 \mathrm{y}$ \\
Mean & $4.5 \mathrm{y}$ \\
Median & \\
Weight (kg) & $4.4-61.4$ \\
Range & 28.3 \\
Mean & 19.8 \\
Median & \\
Indication ( $n$ ) & 10 \\
Oncologic & 5 \\
Infectious/inflammatory & 5 \\
Back pain &
\end{tabular}

${ }^{*}$ Plus a $50-\mathrm{kg}$ 20-y-old. 


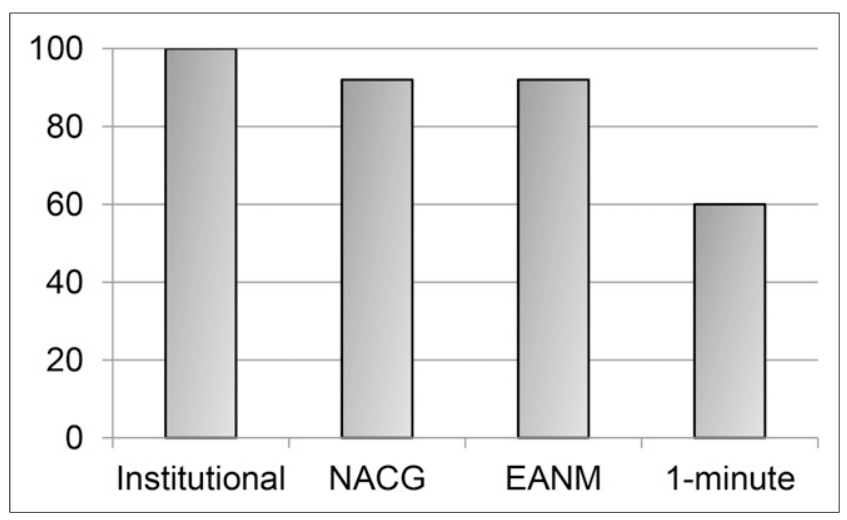

FIGURE 1. Percentage diagnostic by imaging protocol (masked analysis).

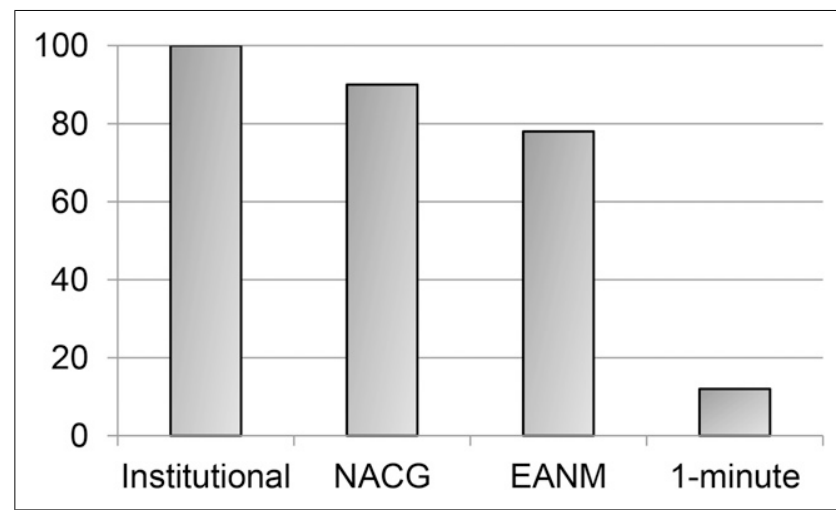

FIGURE 2. Percentage acceptable for daily clinical use (unmasked analysis).

\section{RESULTS}

In our first analysis, the statistical likelihood of producing a diagnostic image with each of the 4 imaging strategies was evaluated. "Diagnostic" was defined as a 2,3 , or 4 on the $1-4$ scale. Separate analysis of the likelihood of producing a "2" versus a " 3 " or "4" was performed, and the results were similar. One hundred percent of the highest-activity images, based on our institution's nomogram, were diagnostic. Ninety-two percent of the NACG and $92 \%$ of EANM images were diagnostic. These values were not significantly different $(P=1$ and 0.08 when NACG images were compared with our institution's images and the EANM images, respectively). Sixty percent of the 1-min images were diagnosticsignificantly fewer than for the other imaging protocols $(P<0.001$ as compared with NACG). These results are graphically summarized in Figure 1.

Four of the 20 patients had pathologic findings based on the interpretations of the contemporaneous diagnostic bone scintigraphy images. All pathologic findings on the institutional, NACG, and EANM images were correctly identified by interpreters. One EANM image was falsely interpreted as having a pathologic finding, and a single 1-min image with a pathologic finding was interpreted as equivocal. Because of the low number of patients with pathologic findings, statistical analysis was not performed on these data, but there were no false-negative results and only a single false-positive result in the institutional, NACG, and EANM
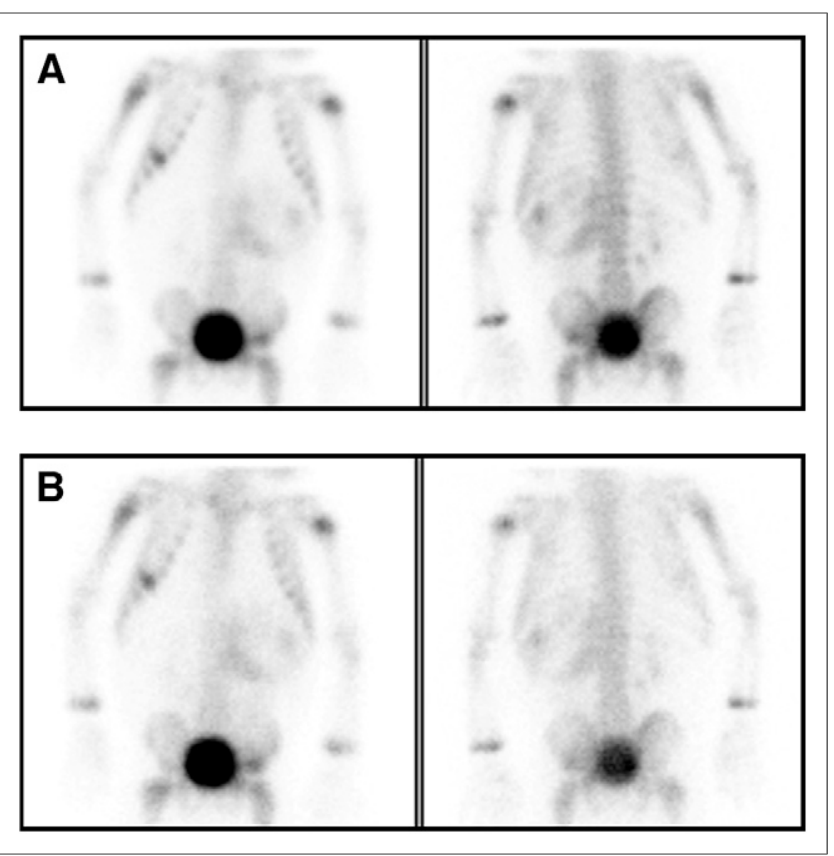

FIGURE 3. Institutional (A) and NACG (B) images of a patient for whom, on unmasked analysis, the institutional images were subjectively deemed acceptable for daily clinical use but the NACG images were not. Abnormal uptake in the abdominal mass and multiple bone metastases is evident on both sets of images.

images. When the lowest-activity 1-min images were considered, there was a single additional false-negative result.

In our second analysis, $100 \%$ of our institution's high-dose images were considered adequate for daily clinical work. This was true of $90 \%$ of the NACG images and $78 \%$ of the EANM images (Fig. 2). Twelve percent of the 1-min images met this criterion. This translated to a slightly statistically significant preference for our institution's images over the NACG images, with a $P$ value of 0.04 . The children for whom the higher-activity images were preferred weighed 4.4 and $13.9 \mathrm{~kg}$. Representative images for these 2 patients are presented as Figure 3. Subanalysis of children over $20 \mathrm{~kg}$ showed no significant difference between high- and low-activity images. The NACG acceptability was not significantly different from the EANM acceptability $(P=0.13)$. The NACG images were statistically more acceptable than the 1-min images, with a $P$ value of less than 0.001 .

\section{DISCUSSION}

It is important to use the lowest amount of radiation possible when imaging patients of all ages, but especially children, for the reasons discussed in the introduction. However, too low a dose can lead to misinterpretations or, ironically, a higher dose if the study has to be repeated. Here, we have presented data that support the lower activities recommended by the NACG. Images representing lower administered activities remained of diagnostic quality, with no statistically significant difference between the NACG images and our institutional images, which required as much as 3 times the radiation exposure. There was a slightly significant preference for the higher-activity images than for the NACG images in the unmasked analysis, based on images of 2 of the 20 patients, who weighed 4.4 and $13.9 \mathrm{~kg}$. Despite this slight preference for the 
images with higher counts, pathologic findings were correctly identified on all institutional, NACG, and EANM images. There were no false-negative examinations with any of the diagnostic protocols, and there was only a single false-positive, with the caveat that the number of patients with pathology within the field of view was low. Taken together, these data support the use of NACG imaging for pediatric bone scintigraphy. For smaller children, particularly those weighing less than $20 \mathrm{~kg}$, the NACG guidelines are acceptable, but ongoing quality assurance should be performed on these patients as they are imaged to ensure that the images are of adequate quality. Images should be evaluated closely before release of the patient from the imaging suite to determine whether additional imaging should be performed. Increasing the imaging time for smaller children should be considered but must be balanced with the potential for increased motion artifacts.

The strengths of this study are the inclusion of a significant number of patients under $20 \mathrm{~kg}$ and the variety of indications for imaging. The major limitation is the small number of patients with pathologic findings. Although there were no false-negatives, the number of patients with pathologic findings was low. Future research in which a greater number of cases have pathologic findings could further investigate whether there is a statistically significant chance that disease will be missed on lower-activity images. Also, we investigated delayed static imaging only. Our data do not include 3-phase studies or studies with SPECT imaging, which are performed much less frequently in pediatric imaging than in adult imaging.

\section{CONCLUSION}

These data validate the use of NACG administered activities for bone scintigraphy. The NACG meet the goal of pediatric dose reduction while maintaining diagnostic quality. For children under $20 \mathrm{~kg}$, the images were less pleasing but still considered diagnostic, and with close quality assurance these smallest children can also be imaged effectively with the lower administered activities.

\section{DISCLOSURE}

The costs of publication of this article were defrayed in part by the payment of page charges. Therefore, and solely to indicate this fact, this article is hereby marked "advertisement" in accordance with 18 USC section 1734 . This project was supported by CTSA award no. UL1TROOO445 from the National Center for Advancing Translational Sciences. Its contents are solely the responsibility of the authors and do not necessarily represent official views of the National Center for Advancing Translational Sciences or the National Institutes of Health. No other potential conflict of interest relevant to this article was reported.

\section{ACKNOWLEDGMENT}

We extend gratitude to Dr. Michael Stabin, who provided the information from ICRP publication 106 that allowed us to calculate effective doses for ${ }^{99 \mathrm{~m}} \mathrm{Tc}$-methylene diphosphonate.

\section{REFERENCES}

1. Dorfman AL, Fazel R, Einstein AJ, et al. Use of medical imaging procedures with ionizing radiation in children: a population-based study. Arch Pediatr Adolesc Med. 2011;165:458-464.

2. Pierce DA, Shimizu Y, Preston DL, Vaeth M, Mabuchi K. Studies of the mortality of atomic bomb survivors. Report 12, Part I. Cancer: 1950-1990. Radiat Res. 1996;146:1-27.

3. Gelfand MJ, Parisi MT, Treves ST. Pediatric radiopharmaceutical administered doses: 2010 North American Consensus Guidelines. J Nucl Med. 2011;52:318-322.

4. Lassmann M, Treves ST; EANM SNMMI Paediatric Dosage Harmonization Working Group. Paediatric radiopharmaceutical administration: harmonization of the 2007 EANM paediatric dosage card (version 1.5.2008) and the 2010 North American consensus guidelines. Eur J Nucl Med Mol Imaging. 2014;41:10361041.

5. Radiation dose to patients from radiopharmaceuticals: a third addendum to ICRP publication 53, ICRP publication 106. Ann ICRP. 2008;38:1-197.

6. Piepsz A, Hahn K, Roca I, et al. A radiopharmaceuticals schedule for imaging in paediatrics. Eur J Nucl Med. 1990;17:127-129.

7. Donohoe KJ, Brown ML, Collier BD, et al. Society of Nuclear Medicine procedure guideline for bone scintigraphy: version 3.0-approved June 20, 2003. http://interactive.snm.org/docs/pg_ch34_0403.pdf. Published August 2003. Accessed July 22, 2015. 